\title{
A case of high-pressure intracerebral pouch
}

\author{
AMIR NAJIM AL-DIN, BERNARD WILLIAMS \\ From the Midland Centre for Neurosurgery and Neurology, Smethwick, England
}

SUMMARY A case is presented of a 13-year-old boy with a long-standing high-pressure pouch in the right parietal lobe containing cerebrospinal fluid. The clinical presentation was of epilepsy. Isotopic studies suggested that the pouch was filling along the subarachnoid space at the back of the corpus callosum in the posterior part of the interhemispheric fissure. There was a dilated sulcus at this point, which filled freely on air encephalography. Pressure studies suggested the likelihood of a venous pulsation filling mechanism with a valvular action. Electrocorticography, excision of an epileptic focus in the wall of the cavity and opening of the ventricular wall into the pouch have produced a good clinical result. Computered tomography after operation has confirmed correction of the high-pressure state.

Cavities containing cerebrospinal fluid (CSF) within the neuraxis may be divided according to various criteria. Normal cavities may be dilated as in hydrocephalus; embryological remnants may be dilated as in hydromyelia; new cavities may be excavated within nervous tissue as in syringomyelia. The occurrence of abnormal cavities in communication with the CSF pathways which are giving rise to pressure symptoms form a particularly interesting group. Terminology is variable: it seems sensible to reserve the term "cyst" for a fluid collection which has no macroscopic connection to other fluidcontaining spaces, and to prefer the term "pouch" for cavities with one entrance or exit. If the cavity has both an entrance and an exit-like a dilated third ventricle-then neither "cyst" nor "pouch" seems appropriate. Dilated pouches under higher pressure than their surroundings occur inside the head or the spine, and may be in any layerextradural, subdural, intra-arachnoid, or subpialand may include diverticula from the ependymal lined surface of the ventricles. This report concerns an unusual case of an intracerebral pouch in communication with the subarachnoid space, with high pressure over a long period, comprehensively studied with a good surgical result.

\section{Case report}

A boy was born in June 1966, weighing $2.55 \mathrm{~kg}$ after a

Address for reprint requests: Bernard Williams, the Midland Centre for Neurosurgery and Neurology, Holly Lane, Smethwick, West Midlands B67 7JX, UK.

Received 10 June 1981, accepted 6 August 1981 prolonged labour. He walked at 11 months, but was enuretic at least once a week up to presentation at the age of 13 years. At the age of 11 he started to have nocturnal generalised seizures once every three months. Six months later he started to have generalised fits during the day: these were preceded by tingling sensation in the left leg and arm but not the face. Sodium valproate was given, and although it reduced the frequency of the fits it did not abolish them.

The patient was an active boy with normal intelligence. His skull was larger on the right side; head circumference was $58.5 \mathrm{~cm}$. The left side of the body was slightly smaller than the right. The left leg was $2 \mathrm{~cm}$ shorter than the right; the left foot had a cavus deformity and was $1 \mathrm{~cm}$ shorter than the right. The cranial nerves, reflexes, coordination and sensation were normal. Both plantar responses were flexor. There were no cranial or cervical bruits. The electoencephalogram was dominated by a mixture of alpha and theta activity. On the right side in the posterior temporal and parietal regions, frequent spike discharges were seen, with phase reversal in the posterior parietal region. Computed tomographic (CT) scan showed a large fluid-filled defect in the right parietal region expanding the vertex of the vault (fig 1). Brain scan following injection of radio-active Indium $\left(\right.$ In $\left.^{111}\right)$ into the cisterna magna showed no radio-activity in the main part of the pouch 26 hours after injection (fig 2). A burr hole was made over the cavity, the dura was left intact to minimise the disturbance to the dynamics of the abnormal CSF collection. An air cystogram showed a cavity not communicating with the ventricles (figs 3,4 ). The fluid had a protein content of $0.13 \mathrm{~g} / \mathrm{l}$. The patient was not disturbed by these investigations, and after seven weeks allowed for the air to absorb, and stable pressure relationships to be re-established, the CT scan appearances were unchanged.

Pressure studies were done with the patient seated and 

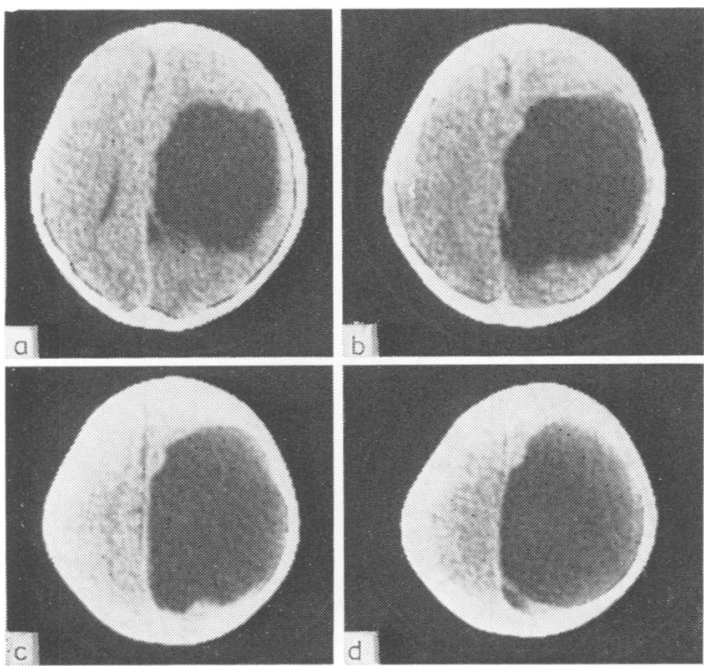

Fig 1 Computed tomographic scans from the level of the ventricles (a) up to almost the vertex of the skull.

Note that there is a membrane visible across the back of the space on (a) and (d): it is partly visible in (b) but is not seen in (c). This was a fenestrated membrane separating the pouch from the subarachnoid space in a distended sulcus.

Fig 3 Lateral radiographs using a horizontal X-ray beam with air in the pouch. Left brow down, right brow up. The extent of the cyst and the expansion of the bone on the right side may be seen.

Fig 2 Emission computed tomographic scans at the level of the cyst 24 hours after cisternal injection of $0.5 \mathrm{mC}$ of In ${ }^{111}$ DPTA. Note that the subarachnoid pathways on the right are not functioning except for the arrowed collection at the back of the pouch corresponding to the distended sulcus. Thin arrows mark the midline; a thick arrow shows the distended sulcus. (View from above)

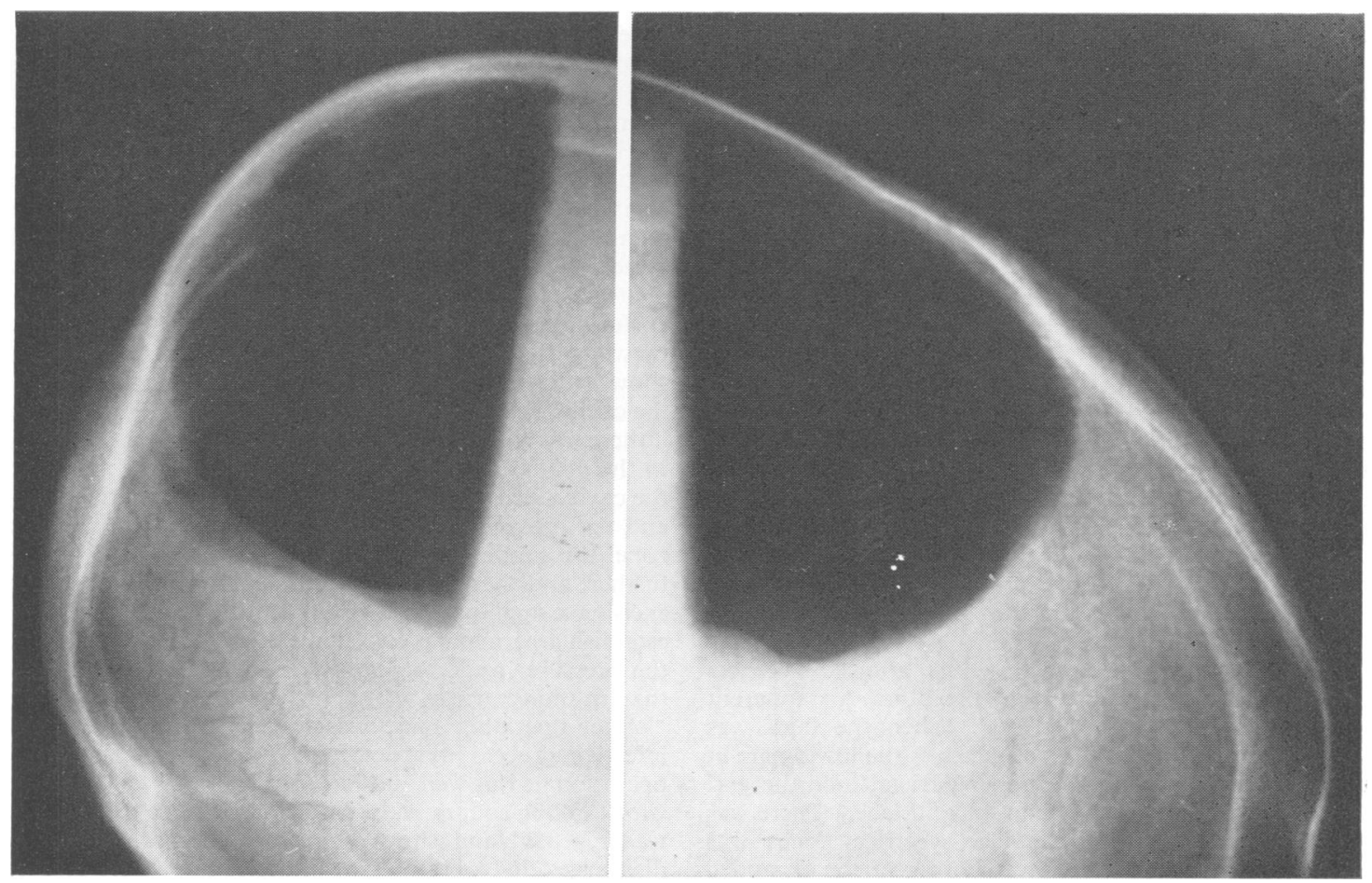




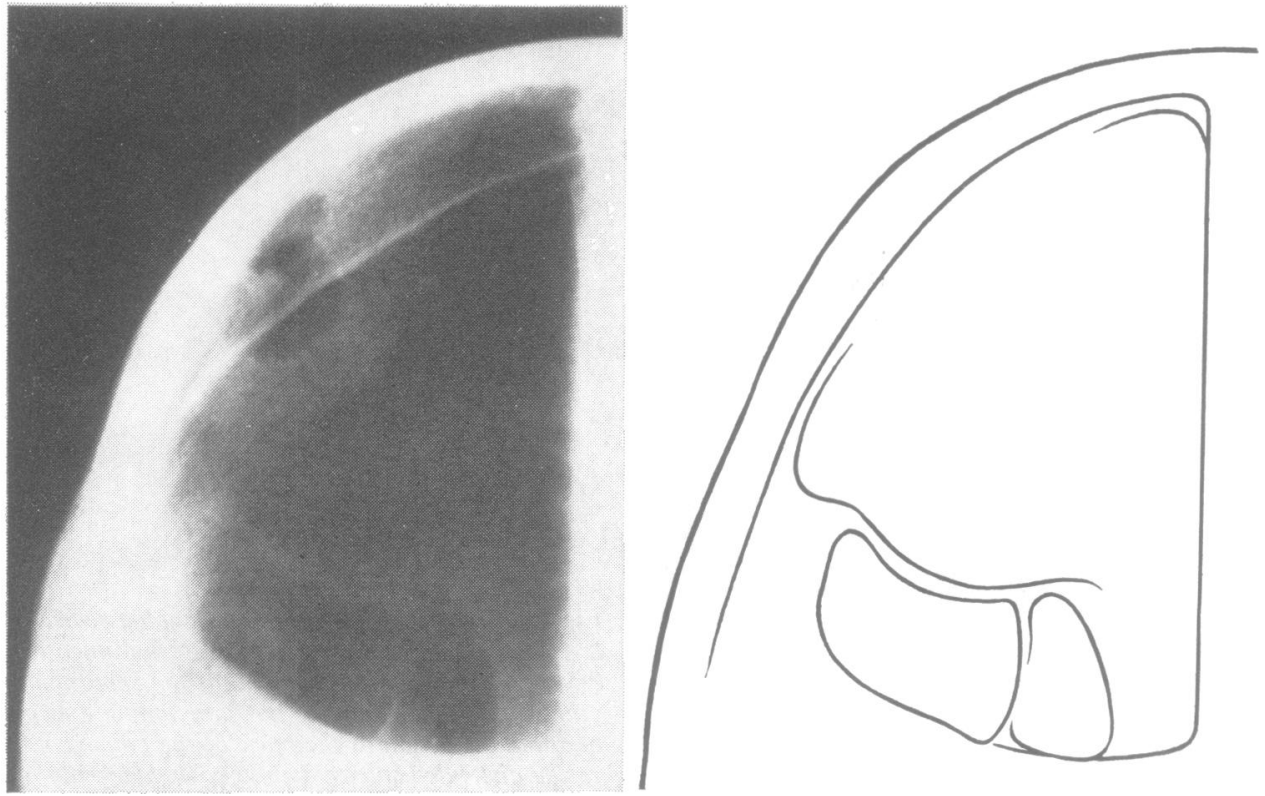

Fig 4 Lateral radiograph brow down with the dilated sulcus seen on an underpenetrated film. The structure is only dimly visible and appears to show the sulcus present in two parts: a drawing is given on the right.

conscious. A needle in the pouch, and another in the lumbar sac, were connected to pressure transducers, and the manometers electrically offset to correct for the differences in height. The cavity pressure was subtracted from the lumbar pressure, giving a differential trace (fig 5). Coughing, straining, Queckenstedt's manoeuvre, and blowing into a mouthpiece, were used in testing. The arterial pulsations were normal with a mean amplitude of $4.2 \mathrm{~mm} \mathrm{Hg}$ in the head and $3.7 \mathrm{~mm} \mathrm{Hg}$ in the spine. Cough pulsations were also within the normal range (30-75 $\mathrm{mm} \mathrm{Hg}$ ) except that the amplitude of the pulsation in the head was only $57 \%$ of that in the spine-normal values for adults being above $70 \%$. Several "blows" were measured with a pressure transducer attached to the mouthpiece into which the patient tried to blow a "square wave" of pressure. The base line pressures were identical throughout the recordings, with the exception of one blow displayed in fig 5. After this there was an apparent change in the base line pressure. After all other manoeuvres the base line pressures were unchanged and remained equal within and outside the abnormal collection.

Operative exposure on 6 May 1980 allowed electrical recordings from the outside of the dura. An apparent epileptogenic focus was located. When the dura was reflected, the brain looked normal over the lower part of the hemisphere, but in the upper parietal lobe the gyri were grossly expanded and the sulci flattened. There was no cystic collection visible. The electrical focus was confirmed as lying at the lower border of the expanded area, and excision of this area included removal of the outer part of the wall of the abnormal cavity at its lower border. The inside of the cavity was seen to be smooth and regular over most of the interior; the wall of the right lateral ventricle protruded into the cavity but was intact. At the back end of the cavity was an area where the white matter appeared to be missing, and the lining of the cavity seemed to be pia mater, through which could be seen a deep and wide sulcus, and beyond it the normal cortex on the opposite wall of the sulcus. This sulcus was dilated and communicated with the cavity by several tiny perforations in the pia. This seemed to be the only route by which fluid could enter the cavity: inspection did not show a definite valve at the orifices; the sulcus outside the cavity was also dilated, and thus it seemed likely that if there was a valvular mechanism present it was proximal to the dilated sulcus in the route by which CSF reached the cavity. Because of its proximity to the calcarine area, and the presence of relatively intact cortex around this zone, the supposed area of the valve was not disturbed further, and the drainage of the pouch was achieved by excising a small part of the wall of the lateral ventricle to establish a permanent communication and to ensure that the pressure inside the cavity could not become higher than in the ventricles.

After operation there was transient pyrexia, but no infective process was detected. Two months after the operation he was well and had had no attacks of epilepsy whilst taking sodium valproate $500 \mathrm{mg}$ twice daily. EEG was repeated and showed none of the focal epileptic discharges that were present in the pre-operative EEGs. There was some delta activity on the right side. CT scans 


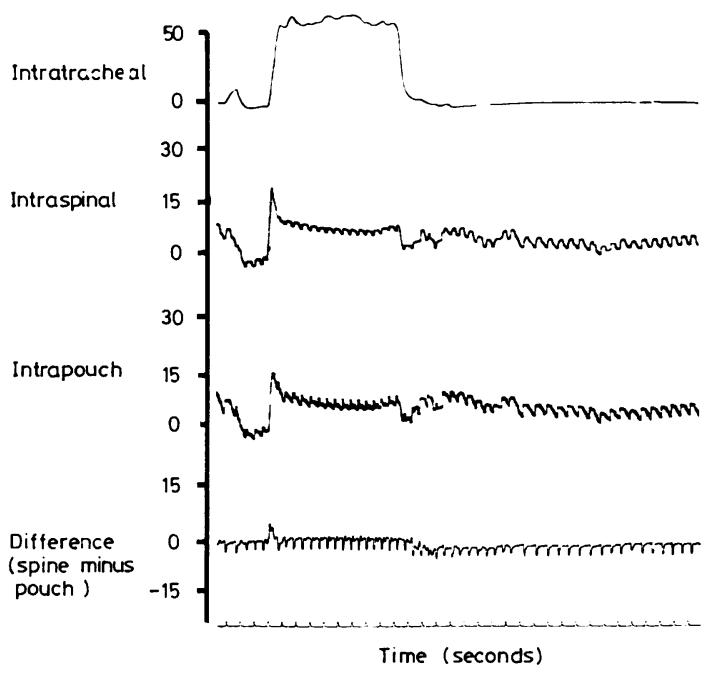

Fig 5 Pressure traces before operation. The vertical scale is in $\mathrm{mmHg}$, and time marked below in seconds, progressing from left to right. The top trace was taken from a pressure transducer on a mouthpiece, and the rise in abdominal and thoracic pressure can be seen during a blow of $10 \mathrm{~s}$. The second trace shows the pressure in a lumbar puncture needle. The third trace is from the pouch, and shows an almost identical pressure increase. The bottom, differential, pressure trace shows that if the pouch pressure is subtracted from the lumbar pressure, a pressure change may be seen after the patient stops blowing. The lumbar pressure drops lower than the pouch pressure, suggesting that a valvular mechanism is at work giving an increase in intrapouch pressure of about $2 \mathrm{mmHg}$.

showed someaccumulation of fluid outside the hemisphere and a loss of tension as shown by relaxed appearance of the walls and diminished volume of the pouch (fig 6).

Histology of the excised area showed the junction of normal brain with thinned-out cortex. The thinned area shown in fig 7 shows axons running parallel with the surface, and no evidence of the normal layers of the cortex.

\section{Discussion}

There are many anatomical sites in which intracranial pouches of this kind may have their openings. The posterior fossa is a common site, with the opening close to the foraman magnum. ${ }^{1-3}$ Another common site is the incisura ${ }^{4-6}$ or in the mouth in the Sylvian fissure. ${ }^{7-9}$ An opening in the subarachnoid space of the central fissure is uncommon. ${ }^{510}$

The clinical and radiographic features of this case leave no doubt that this was a high-pressure pouch. The differentialyand overall expansion of the skull, the displacement of the ventricles, and the globular

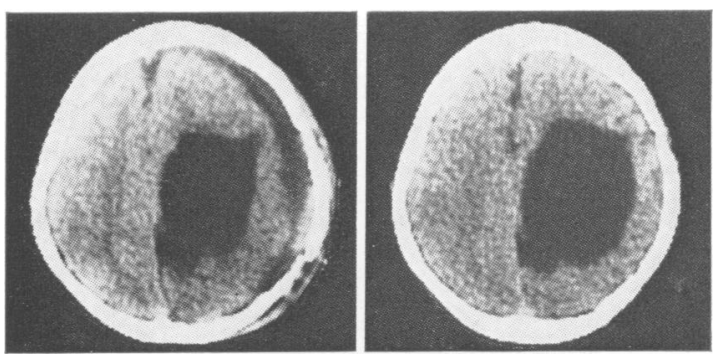

Fig 6 Computed tomographic scans two weeks after operation, showing fluid over the surface of the hemisphere and shrinkage of the pouch which has lost its globular tendency.

outline of the pouch all indicate this, and the clinical course and complaints support the contention. The increase of cerebral bulk after operation offered a striking illustration of the correction of a previous high-pressure state.

The assumption that the fluid was CSF and entered the pouch along the subarachnoid spaces at the back of the central fissure seems reasonable. The filling of the pouch was almost certainly intermittent, and the failure to demonstrate isotope in the main part of the pouch is consistent with this assumption. The pouch, being under raised pressure, occluded the CSF pathways generally on the right side of the head. The amount of CSF entering the pouch was probably small when compared with the flow through the normal subarachnoid pathways: the high pressure resulted because the rate of absorption from the pouch was slow. Activity in the bulk of the pouch was not discernibly above the background activity in the emission computed tomography scan. The filling of the sulcus is however clearly seen. This passageway in the midline was also visible in the rectilinear scans. For a description of the emission tomography method used, see Woolley, Williams and Venkatesh. ${ }^{11}$ Even if several millilitres of isotope labelled CSF had entered the main pouch during the 48 hours or so in which there was significant activity, the dilution was so great that it is likely that it would be imperceptible below the background radioactivity.

Pressure within the cavity higher than that within the ventricles means that fluid cannot flow into it from the ventricles and subarachnoid space by passive flow. There must be a transient pulsatile phenomenon which pumps the fluid from a normally low pressure zone into the cavity, and then some form of valvular action which prevents its immediate return. This has been apparent to many who have contributed to the literature on such cysts, and the most popular explanation has been that some derivation of arterial energy has been at work, ${ }^{7} 91213$ 


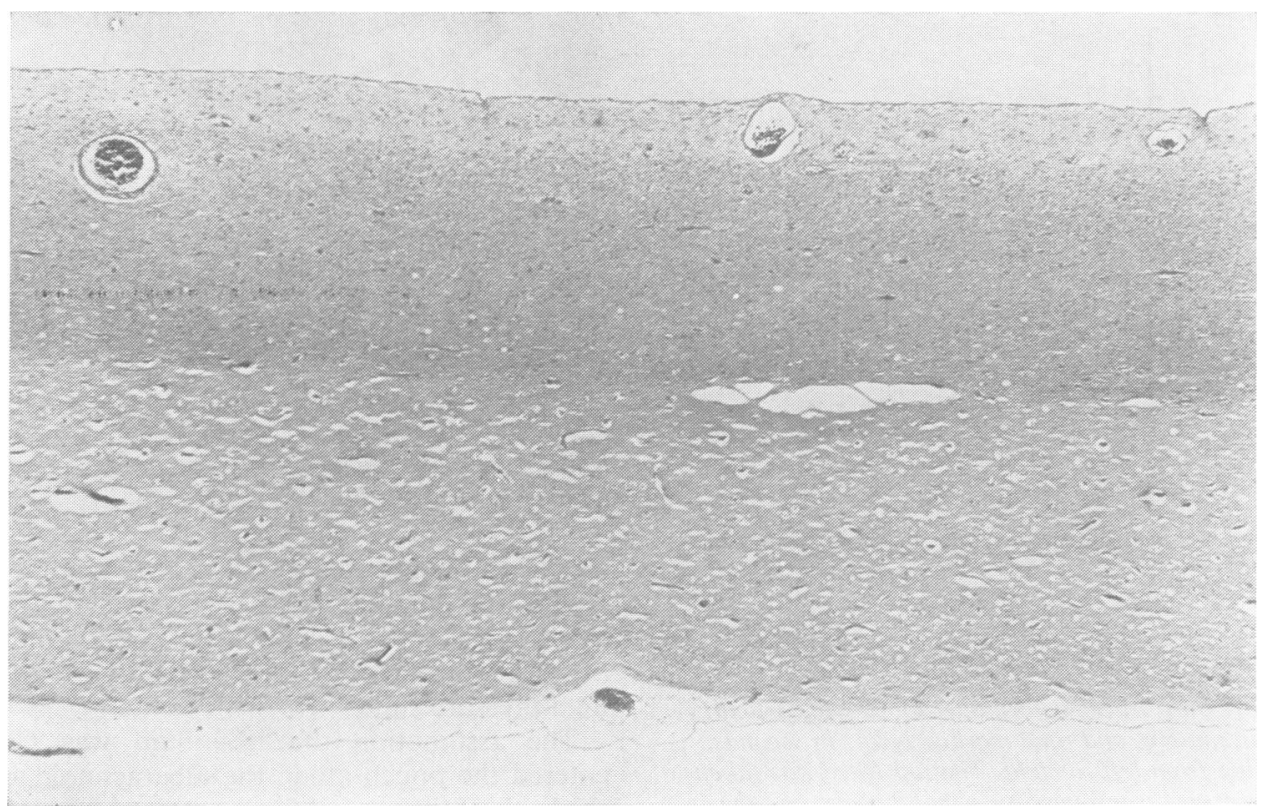

Fig 7 Low-power view of the thinned area over the pouch. The arachnoid shown at the top is closely applied and the sulci are so flattened that their previous site is visible only by the situation of the vessels. There was no evidence of layering of cortical neurones, the axons being disposed parallel to the brain surface. The white matter showed normally myelinated axons deficient in numbers. The innermost zone is entirely gliotic. There is a lining on the inside of the pouch of flattened cells, probably glial.

Direct transmission of pulsatile energy from the arterial wall to the CSF seems unlikely, and has been criticised by Williams and Guthkelch. ${ }^{14}$ The possible pumping action by movement of the brain parenchyma in response to the systolic pulse wave is more probable. It can be seen to be responsible for movement of fluid within the aqueduct, for example. ${ }^{13}$ This movement in the aqueduct is largely caused by narrowing from side to side of the third ventricle during systole, and such pulsation is also likely to occur in other sites where the CSF containing spaces are extensive but narrow. Thus the capillary bed of the brain parenchyma may be responsible for pulsation in the basal cisterns, the Sylvian fissure or the interhemispheric fissure. Arterial pulsations are small in comparison to the large pressure swings and accompanying movements which result from changes in venous pressure after such events as coughing, sneezing or straining. Studies of such pressure swings by Williams ${ }^{14-19}$ have led to the suggestion that pressures transmitted through the nervous system are likely to be responsible for progression of some severe deformities of the neuraxis, such as syringomyelia and hindbrain hernia, as well as subarachnoid pouches.

The occurrence of a pouch in this situation is uncommon, but it is notable that in some cases of hydrocephalus, particularly in association with posterior fossa tumour, fluid tends to flow up the back part of the interhemispheric fissure. This may be verified on isotope scanning after isotopic studies following CSF injections. Dilation of the posterior callosal pathways in this situation has also been described as a radiographic finding by Schechter, Bull and Carey. ${ }^{20}$ They likened the appearances to those of "rabbit's ears" and suggested that dilation of this part of the pathway was due to a "circling back" of the arterial pulse. It seems more probable, in view of recent studies of the venous pulse and demonstration of its upward thrust, that it is this pulsation which is responsible for dilating the subarachnoid pathways in the posterior part of the interhemispheric sulcus.

Whatever normally thrusts fluid into this part of the system almost certainly thrusts it a little further in the case under discussion: presumably dilated one sulcus in particular, and therefore causing the CSF to dissect into the white matter. As the pouch expanded, it acted as a space-occupying lesion, further compressing the surface pathways, especially on the right, and continuing to divert the CSF up the abnormal posterior callosal pathway. The 
presence of a space-occupying lesion and accompanying high pressure also is likely to aggravate the amplitude of pulsation by diminishing the capacitance of the neuraxis. ${ }^{15}$ This increase in pulsation was not proven in this case: neither was the filling of the cyst certainly demonstrated, because the recording is not free from the suspicion of artefact, and until movement can be demonstrated at the same time as pressure change, the proof of inflation by this mechanism has not been provided. The illustration in fig 5 nevertheless suggests strongly an influx of CSF into the cavity during blowing, and our belief is that energy derived from the contraction of muscles around the body cavities and transmitted to the CSF by epidural veins is the most likely cause. Relatively simple surgical correction of the deformities, allowing pressure differences to be maintained, has produced a satisfactory cure.

We are grateful to Dr CD Thursfield and Miss Angella Bryan for pressure recordings; and to Eddie Lowe for help with photography. This patient was referred by Dr RC Hughes.

\section{References}

${ }^{1}$ Kaplan A. Pia-arachnoidal cysts of the posterior fossa. Am J Surg 1948;76:102-6.

${ }^{2}$ Gilles FH, Rockett FX. Infantile hydrocephalus: retrocerebellar "arachnoidal" cyst. J Pediatr 1971; 79:436-43.

${ }^{3}$ Leschey WH. Posterior fossa arachnoidal cysts. $J$ Maine Med Assoc 1979;70:398-405.

- Faris AA, Bale GF, Cannon B. Arachnoidal cysts of the third ventricle with precocious puberty. South Med J 1971;64:1139-42.

${ }^{5}$ Harrison MJG. Cerebral arachnoid cysts in children. J Neurol Neurosurg Psychiatry 1971;34:316-23.
${ }^{6}$ Williams B, Guthkelch AN. Expansion of central arachnoid pouches. Child's Brain 1975;1:364-7.

7 Dott NM, Gillingham FJ. Mechanical aspects of the cerebrospinal fluid circulation-physiological, pathological, surgical. In: Wolstenholme GEW, O'Connel CM, eds. Cerebrospinal Fluid. London: Churchill, 1958:246-64.

${ }^{8}$ O'Connell JE. Cerebrospinal fluid mechanics. Pro Roy Soc Med 1970;63:507-18.

${ }^{9}$ Starkman SP, Brown TC, Linell EA. Cerebral arachnoid cysts. $J$ Neuropath Exp Neurol 1958;17:484-500.

10 Jabubiak P, Dunsmore RH, Beckett RS. Supratentorial brain cysts. $J$ Neurosurg 1968;28:129-36.

1 Woolley J, Williams B, Venkatesh S. Cranial isotopic section scanning. Clin Radiol 1977;28:517-28.

${ }^{12}$ Du Boulay GH. Pulsatile movements in the cerebrospinal fluid pathways. Brit J Radiol 1966;39:255-62.

${ }^{13}$ Du Boulay GH, O'Connell JE, Currie J, Bostick T, Verity $P$. Further investigations on pulsatile movements in the cerebrospinal fluid pathways. Act Radiol 1972;13:496-523.

14 Williams B, Guthkelch AN. Why do central arachnoid pouches expand? J Neurol Neurosurg Psychiatry 1975;37:1085-92.

15 Williams B. A demonstration analogue for ventricular and intraspinal dynamics. J Neurol Sci 1974;23: 445-61.

${ }^{16}$ Williams B. Cerebrospinal fluid pressure differentials in spina bifida cystica with special reference to the Arnold-Chiari malformation. J Dev Med Child Neurol 1975;35:138-50.

17 Williams B. Cerebrospinal fluid pressure changes in response to coughing. Brain 1976;99:331-46.

18 Williams B. Subarachnoid pouches of the posterior fossa with syringomyelia. Acta Neurochir 1979;47: 187-217.

19 Williams B. Cough headache due to cerebrospinal pressure dissociation. Arch Neurol 1980;37:226-30.

${ }^{20}$ Schechter NN, Bull JWD, Carey P. Two new encephalographic signs of pressure hydrocephalus. Brit $J$ Radiol 1958;31:317-25. 